\title{
Pathophysiological differences betweenmultifocal fibromuscular dysplasia and atherosclerotic renal artery stenosis
}

Citation for published version (APA):

van Twist, D. J. L., Houben, A. J. H. M., de Haan, M. W., de Leeuw, P. W., \& Kroon, A. A. (2017). Pathophysiological differences betweenmultifocal fibromuscular dysplasia and atherosclerotic renal artery stenosis. Journal of Hypertension, 35(4), 845-852. https://doi.org/10.1097/HJH.0000000000001243

Document status and date:

Published: 01/04/2017

DOI:

10.1097/HJH.0000000000001243

Document Version:

Publisher's PDF, also known as Version of record

Document license:

Taverne

Please check the document version of this publication:

- A submitted manuscript is the version of the article upon submission and before peer-review. There can be important differences between the submitted version and the official published version of record.

People interested in the research are advised to contact the author for the final version of the publication, or visit the DOI to the publisher's website.

- The final author version and the galley proof are versions of the publication after peer review.

- The final published version features the final layout of the paper including the volume, issue and page numbers.

Link to publication

\footnotetext{
General rights rights.

- You may freely distribute the URL identifying the publication in the public portal. please follow below link for the End User Agreement:

www.umlib.nl/taverne-license

Take down policy

If you believe that this document breaches copyright please contact us at:

repository@maastrichtuniversity.nl

providing details and we will investigate your claim.
}

Copyright and moral rights for the publications made accessible in the public portal are retained by the authors and/or other copyright owners and it is a condition of accessing publications that users recognise and abide by the legal requirements associated with these

- Users may download and print one copy of any publication from the public portal for the purpose of private study or research.

- You may not further distribute the material or use it for any profit-making activity or commercial gain

If the publication is distributed under the terms of Article $25 \mathrm{fa}$ of the Dutch Copyright Act, indicated by the "Taverne" license above, 


\title{
Pathophysiological differences between multifocal fibromuscular dysplasia and atherosclerotic renal artery stenosis
}

\author{
Daan J.L. van Twist ${ }^{a, b}$, Alfons J.H.M. Houben ${ }^{a, b}$, Michiel W. de Haan $^{c}$, Peter W. de Leeuw ${ }^{a, b, d}$, \\ and Abraham A. Kroon ${ }^{a, b}$
}

Background: Fibromuscular dysplasia (FMD) and atherosclerotic renal artery stenosis (ARAS) are the most common causes of renovascular hypertension. So far, FMD is believed to cause hypertension via similar mechanisms as in ARAS, that is, a decrease in renal blood flow, which subsequently leads to increased renin secretion. However, given the differences in the blood pressure (BP)-lowering effect of revascularization between patients with ARAS and FMD, we questioned whether this is true.

Methods: We measured renal blood flow ( ${ }^{133}$ Xenon washout method) and renin secretion per kidney and their relationship to BP in a cohort of 64 patients with multifocal FMD and 110 patients with ARAS (off medication, prior to revascularization).

Results: We found that renal blood flow is significantly higher in FMD as compared with ARAS. In patients with unilateral ARAS, renin secretion was increased in the affected kidney as compared with the unaffected kidney. This lateralization in renin secretion, however, was not found in unilateral FMD. After correction for differences in baseline characteristics, we found that systemic renin levels and local renin secretion was lower in FMD as compared with ARAS. Moreover, the relationship between BP and renin secretion in FMD was inverse to that in ARAS.

Conclusion: These findings argue against the hypothesis that FMD induces hypertension via similar pathophysiological mechanism as in ARAS.

Keywords: fibromuscular dysplasia, hypertension, renal artery stenosis, renal blood flow, renin, renin-angiotensin system, renovascular hypertension

\begin{abstract}
Abbreviations: 24-hABPM, 24-h blood pressure measurement; 2K1C, two-kidney-one-clip; APRC, active plasma renin concentration; ARAS, atherosclerotic renal artery stenosis; AU, arbitrary units; CKD-EPI, Chronic Kidney Disease Epidemiology Collaboration; eGFR, estimated glomerular filtration rate; FMD, fibromuscular dysplasia; MAP, mean arterial blood pressure; MRBF, mean renal blood flow; RSR, renin secretion rate
\end{abstract}

\section{INTRODUGTION}

$\mathrm{F}$ ibromuscular dysplasia (FMD) is the second-most common cause of renovascular hypertension [1]. In contrast to atherosclerotic renal artery stenosis
(ARAS), little is known about the pathophysiological mechanisms causing hypertension in these patients, a fact that was recently highlighted in scientific statements of the European Society of Hypertension and the American Heart Association [1,2]. Until now, renal artery FMD is believed to cause hypertension through a decrease in renal blood flow, which subsequently leads to increased renin secretion [3], similar as in two-kidney-one-clip (2K1C) animal models and in patients with renovascular hypertension caused by ARAS. Recently, we challenged this hypothesis by demonstrating that renin secretion and renin-angiotensin system activity are not increased in kidneys with FMD, but comparable with that in kidneys from patients with essential hypertension [4]. Hence, as several characteristics, such as age, sex, and the response to revascularization, substantially differ between patients with FMD and patients with ARAS [1], we questioned whether the commonly held (but unproven) hypothesis that FMD causes hypertension via similar mechanisms as in patients with ARAS is true. Therefore, we studied renal blood flow, renin secretion, and their relationship to blood pressure (BP) in a cohort of patients with untreated multifocal renovascular FMD and compared these with those in patients with ARAS.

\section{METHODS}

\section{Patients}

We prospectively collected data from all hypertensive patients who were angiographically evaluated for the presence of renovascular abnormalities in our hospital during the past two decades. These patients had been referred because of difficult-to-treat hypertension (e.g. BP remaining above goal despite the use of three full-dose antihypertensive drugs) and/or clinical suspicion of renovascular abnormalities (e.g. presence of an abdominal bruit,

Journal of Hypertension 2017, 35:845-852

${ }^{a}$ Department of Internal Medicine, ${ }^{b}$ Cardiovascular Research Institute Maastricht (CARIM), 'Department of Radiology, Maastricht University Medical Center (MUMC+), Maastricht and dDepartment of Internal Medicine, Zuyderland Medical Center, Sittard, The Netherlands

Correspondence to Prof Dr Abraham A. Kroon, Department of Internal Medicine, Maastricht University Medical Center, PO Box 5800, 6202 AZ Maastricht, The Netherlands. Tel: +31 433877005; fax: +31 433875006; e-mail: aa.kroon@mumc.nl

Received 3 October 2016 Revised 30 November 2016 Accepted 7 December 2016 J Hypertens 35:845-852 Copyright (C) 2017 Wolters Kluwer Health, Inc. All rights reserved.

DOI:10.1097/HJH.0000000000001243 
peripheral vascular disease, or a decrease in renal function following treatment with an angiotensin-converting enzyme inhibitor or angiotensin II type-1-receptor blocker).

\section{Protocol}

To avoid interference with the experiments, patients discontinued all antihypertensive medication 3 weeks before the angiographic evaluation. Patients with high risk for acute cardiovascular events [i.e., a recent cardiovascular event $(<6$ months) or an unacceptable large rise in $\mathrm{BP}$ after discontinuation of antihypertensive drugs] were excluded from the study. The day preceding the angiographic evaluation, patients underwent 24-h BP measurement [24-h ABPM, using SpaceLabs ambulatory BP monitor type 90207 or $90217 \mathrm{~b}$ (Spacelabs Healthcare, Snoqualmie, Washington, USA)] and collected a 24-h urine specimen for measurement of sodium excretion as a marker for dietary sodium intake [5].

Patients were admitted to our ward and were fasted overnight. Under continuous monitoring of heart rate and BP (intra-arterially), we selectively catheterized the aorta and both renal veins via the femoral route and drew blood samples from the aorta and both renal veins. After this, we measured mean renal blood flow (MRBF, expressed as $\mathrm{ml} / 100 \mathrm{~g}$ kidney/min) using the ${ }^{133}$ Xenon washout technique as previously described [6]. In short, ${ }^{133}$ Xenon is infused directly into the renal artery, and the washout of ${ }^{133}$ Xenon is recorded with a scintillation counter positioned extracorporeally at the kidney level. Exhaled ${ }^{133}$ Xenon is captured via a tight mask and led through a charcoal filter. After subtraction of background radiation, the washout of ${ }^{133}$ Xenon from the kidney is analyzed mathematically using an exponential decay model. From this model, MRBF is calculated. In the majority of patients, MRBF was measured twice in each kidney. The mean of the two measurements was used for the analyses. In our hospital, ${ }^{133}$ Xenon washout technique has a variation coefficient of $8 \%$ for repeated measurements [6]. Following the ${ }^{133}$ Xenon washout procedure, we performed diagnostic subtraction angiography of the renal arteries. No contrast material was administered prior to completion of the blood sampling and ${ }^{133}$ Xenon washout studies.

Selective renal angiography was performed with injection of 12-ml iopromide (Ultravist 300; Bayer HealthCare; Berlin, Germany) through a 5 French end-hole Cobra-2 or Simmons-2 catheter (Cordis; Cardinal Health, Dublin, Ohio, USA). Angiographic images of the abdominal aorta and renal arteries were obtained in anterior-posterior and two oblique views using a 4 French Universal Flush catheter (Cordis; Cardinal Health) positioned at the level of the renal arteries and injection of $25 \mathrm{ml}$ of iopromide. If clinically indicated, balloon angioplasty was performed. The angiographic films were analyzed by two experienced, independent radiologists who were unaware of the results of the MRBF measurements. The patients were classified as having either no renal artery abnormalities, ARAS (unilateral or bilateral), or FMD (unilateral or bilateral), depending upon the presence or absence of visible abnormalities in the renal artery. In the case of ARAS, the radiologists noted the degree of the stenosis (low grade less than 50\% reduction in luminal diameter, high grade more than
50\%), which was measured using an electronic caliper. As obtaining an objective measure of stenosis is difficult in the case of FMD, the degree of stenosis was not calculated in these patients. In the case of disagreement on renal artery abnormalities, a third radiologists' opinion was decisive. This protocol was approved by the Medical Ethics Committee of the Maastricht University Medical Centre, and all patients gave written informed consent.

\section{Inclusion criteria}

Patients were included in the present analysis when both a complete renal angiography and selective blood samples were available. We included all patients who were diagnosed with FMD of the multifocal type, which is defined as the presence of more than two nonatherosclerotic stenoses with a typical string-of-beads appearance in at least one renal artery [7]. The majority of these patients were also included in a previous study on renal hemodynamics in FMD [4]. Furthermore, we included patients with highgrade ARAS, which was defined as a reduction in luminal diameter of $50 \%$ or more. Patients with unifocal FMD of the renal artery were excluded, as previous data suggest that unifocal lesions probably represent a different disease entity $[7,8]$ and difficulties could arise in distinguishing these from ARAS. Patients with low-grade ARAS (a reduction in luminal diameter of less than $50 \%$ ) were also excluded, as these are usually considered to be of no hemodynamic significance. In addition, we excluded patients in whom renovascular abnormalities had been previously treated, patients with a combination of ARAS and FMD, patients with other secondary causes of hypertension, and patients who had only one kidney (congenital or acquired), a contracted kidney, a tumor of the kidney, syndromal disease (such as Ehlers-Danlos syndrome or Marfan syndrome), or inflammatory vascular diseases (e.g. Takayasu or giant-cell arteritis).

\section{Calculations and analyses}

We measured active plasma renin concentration (APRC) in blood samples obtained from the renal artery and both renal veins. APRC [9] and aldosterone levels (Coat-A-Count Radioimmunometric Assay, Siemens; Los Angeles, California, USA) were measured using an immunoradiometric assay. We assessed the renal renin data in three different ways according to previous literature: renin (V-A)/A, calculated as the venous-arterial difference in APRC divided by the arterial APRC [10]; renal vein renin ratio (renal vein renin level of the affected kidney divided by renal vein renin level of the nonaffected kidney); and [11] renin secretion rate (RSR) [12], calculated as the product of the venous-arterial difference in APRC and the renal plasma flow [MRBF $\times(1-$ hematocrit $)]$, a method that makes it possible to calculate the net renin production per $100 \mathrm{~g}$ of kidney tissue for each individual kidney.

Renovascular resistance (RVR) was calculated as mean arterial BP divided by MRBF and is expressed as arbitrary units. Estimated glomerular filtration rate (eGFR) was calculated using the Chronic Kidney Disease Epidemiology Collaboration formula [13]. We performed a post-hoc analysis to evaluate the BP response to balloon angioplasty 
in patients with FMD. This was done to determine whether the string-of-beads truly played a role in causing high BP or merely was an 'innocent bystander' (i.e., the patients had essential hypertension instead). According to previous literature (the 'Rundback criteria') [14], a response was defined as a reduction in DBP at least $15 \mathrm{mmHg}$, an SBP $140 \mathrm{mmHg}$ or less, and/or a DBP $90 \mathrm{mmHg}$ or less (mean of the second and last measurement of three office BP measurements with 1-min intervals) 1 year after balloon angioplasty without an increase in the number of antihypertensive drugs (evaluated using WHO's Defined Daily Doses classification) [15]. Another post-hoc analysis was performed in patients with ARAS to evaluate whether changing the cutoff from more than 50 to more than $70 \%$ reduction in luminal diameter would influence the results. SPSS (SPSS Inc., version 24.0; Chicago, Illinois, USA) and Graphpad (Graphpad Software Inc., version 5.01; San Diego, California, USA) were used for the statistical analyses. To increase statistical power for analyses of correlations of the outcome measures, we pooled the data obtained from affected kidneys of patients with unilateral ARAS or FMD with the data obtained from the right kidney of patients with bilateral ARAS or FMD, respectively. $t$ tests (paired and unpaired) were used for normally distributed data (expressed as mean \pm SD unless otherwise stated). We used Wilcoxon signed rank test and Mann-Whitney $U$ test for nonnormally distributed data (expressed as medians and interquartile range). Chi square test was used to assess categorical data, and correlation was tested using Pearson's $R$. An analysis of covariance (ANCOVA) model was used to adjust for preexisting differences in patient characteristics that are known to be of influence on the outcome data. A $P$ value less than 0.05 was considered statistically significant.

\section{RESULTS}

\section{Patient characteristics}

In total, 64 patients with multifocal FMD and 110 patients with ARAS (reduction in luminal diameter of at least 50\%) were included (all white). As shown in Table 1, several differences in patient characteristics existed between ARAS

TABLE 1. Characteristics of the patients

\begin{tabular}{|c|c|c|}
\hline Patient characteristic & FMD & ARAS \\
\hline$N$ & 64 & 110 \\
\hline Sex (male/female) & $14 / 50$ & $64 / 46^{*}$ \\
\hline Age (years) & $53.8 \pm 11.0$ & $63.5 \pm 7.9^{*}$ \\
\hline BMI $\left(\mathrm{kg} / \mathrm{m}^{2}\right)$ & $26.7 \pm 4.6$ & $26.5 \pm 4.0$ \\
\hline Diabetes mellitus $[n(\%)]$ & $2(3 \%)$ & $17(16 \%)^{* *}$ \\
\hline Current smoking $[n(\%)]$ & $13(20 \%)$ & $40(36.4 \%)^{*-}$ \\
\hline 24-h SBP (mmHg) & $154 \pm 26$ & $170 \pm 23^{* *}$ \\
\hline 24-h DBP (mmHg) & $91 \pm 16$ & $92 \pm 13$ \\
\hline eGFR $\left(\mathrm{ml} / \mathrm{min}\right.$ per $\left.1.73 \mathrm{~m}^{2}\right)$ & $79 \pm 23$ & $54 \pm 23^{*}$ \\
\hline $\begin{array}{l}\text { Urinary sodium excretion } \\
\text { (mmol/24h) }\end{array}$ & $95 \pm 60$ & $94 \pm 60$ \\
\hline
\end{tabular}

Characteristics of the study patients. Values expressed as mean \pm SD. ARAS,

atherosclerotic renal artery stenosis; eGFR indicates estimated glomerular filtration rate using the Chronic Kidney Disease Epidemiology Collaboration formula [13]; FMD,

fibromuscular dysplasia.
${ }^{*} P<0.001$ vs. FMD.

${ }^{* *} P<0.05$ vs. FMD. and FMD: patients with FMD were more often women $(P<0.001$ vs. ARAS $)$, were younger $(P<0.001)$, had a lower prevalence of smoking and diabetes mellitus $(P<0.05)$, and had a higher eGFR $(P<0.001)$. A total of 24 -h SBP was significantly higher in ARAS $(P=0.002 ; 24$-h ambulatory BP, data available for 62 patients with ARAS and 35 patients with FMD). No differences were found for 24-h urinary sodium excretion. During the study period, no acute cardiovascular events were reported, and none of the study participants had to be excluded from the study because of unacceptably high BP.

\section{Renal blood flow}

As shown in Fig. 1, MRBF of both kidneys was significantly higher in FMD as compared with ARAS $(P<0.001)$, both in patients with unilateral abnormalities and in patients with bilateral abnormalities. No significant differences in MRBF were found between the affected and nonaffected kidney in

(a)

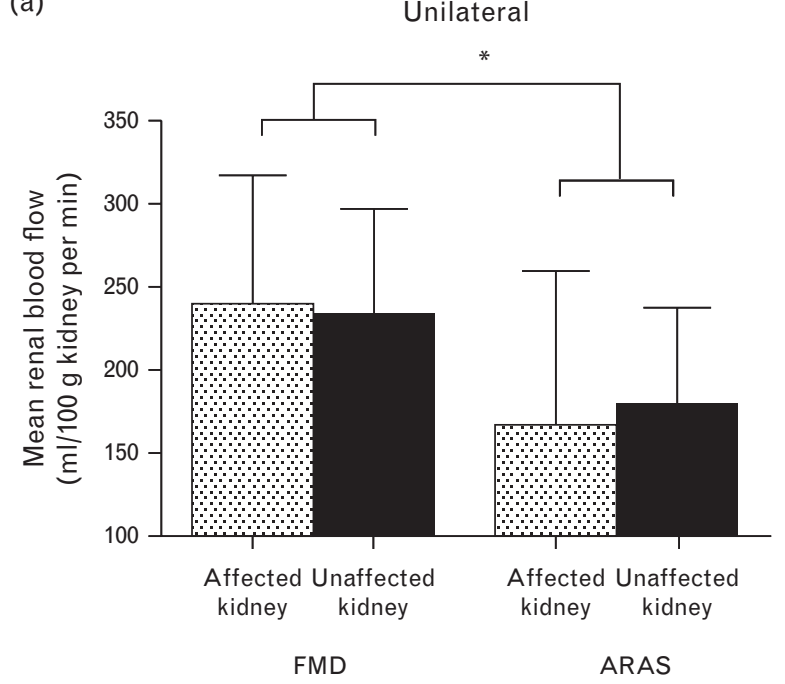

(b)

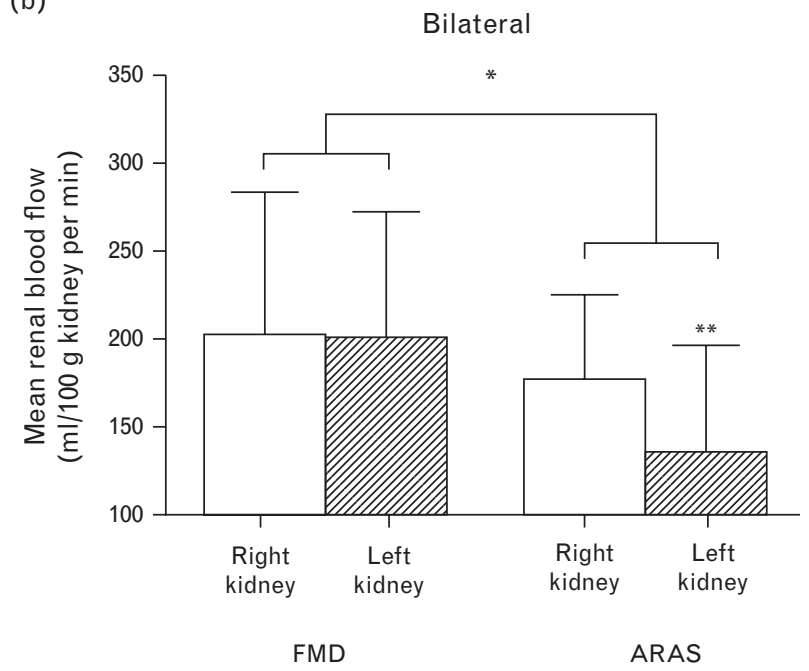

FIGURE 1 Mean renal blood flow. Mean renal blood flow according to the presence of renovascular abnormalities, split up for unilateral (a) or bilateral (b) renovascular abnormalities. Values expressed as mean \pm SD. ARAS, atherosclerotic renal artery stenosis; FMD, fibromuscular dysplasia. ${ }^{*} P<0.001$ vs. fibromuscular dysplasia, ${ }^{* *} P=0.003$ vs. right kidney. 
unilateral FMD or ARAS, but in patients with bilateral ARAS, MRBF of the left kidney was significantly lower as compared with the right kidney $(P=0.003)$. In FMD, however, no such difference was observed. From 10 patients with FMD and 22 patients with ARAS, MRBF could not be obtained due to technical problems with the administration of ${ }^{133}$ Xenon (difficulties in advancing the catheter into the renal artery or patient's intolerance of wearing the mask that prevents spreading of exhaled ${ }^{133}$ Xenon into the room). As shown in Table 2, RVR was significantly higher in kidneys with ARAS as compared with FMD.

\section{Renin}

We found a wide variation in systemic renin levels, but without significant differences between the groups (Table 2). Moreover, no differences in aldosterone levels were observed (Supplemental Table S1, http://links.lww.com/ HJH/A718). Renin (V-A)/A (Table 2) and RSR (Fig. 2) were comparable between bilateral FMD and bilateral ARAS, without differences between the left and right kidney. Out of 64 patients with unilateral ARAS, renal vein renin ratio (cutoff $\geq 1.5$ based on previous literature) [11] was positive in 20 patients (mean of all patients with unilateral ARAS 1.55, range $0.70-6.00$ ) and renin $(\mathrm{V}-\mathrm{A}) / \mathrm{A}$ (cutoff $\geq 0.48$ based on previous literature) [10] was positive in 19 patients (Table 2). In unilateral FMD, however, renal vein renin ratio (mean 0.98 , range $0.72-1.22 ; P=0.002$ vs. ARAS) and renin (V-A)/A were below cutoff in all 36 patients. In unilateral ARAS, RSR and renin (V-A)/A were significantly higher in the affected kidney as compared with the nonaffected kidney $(P=0.014$ and $P<0.001$, respectively). In unilateral FMD, no differences were observed between both kidneys. Renin (V-A)/A in the affected kidney was significantly higher in unilateral ARAS as compared with unilateral FMD $(P=0.032)$. However, after correction for renal blood flow by calculating the RSR, this difference is no longer statistically significant (Fig. 2).

\section{Adjusted outcomes and post-hoc analyses}

As several differences in patient characteristics existed between the groups, we adjusted the outcome measures for age, sex, and 24-h urinary sodium excretion using an ANCOVA model. For these analyses, data obtained from the affected kidneys of patients with unilateral ARAS or FMD were pooled with the data obtained from the right kidney of patients with bilateral ARAS or FMD, respectively. The difference in MRBF between FMD and ARAS was still statistically significant $(206 \pm 27$ vs. $183 \pm 23 \mathrm{ml} / 100 \mathrm{~g}$ kidney/min, respectively, $P<0.001)$. Moreover, arterial renin levels [26.8 (20.8-41.3) vs. $39.7(26.2-54.4) \mathrm{mU} / 1, P<0.05]$ and RSR $[0.35(0.02-0.81)$ vs. $1.03(0.68-3.34) \mathrm{mU} / 100 \mathrm{~g}$ kidney/min, $P<0.05]$ were after adjustment significantly lower in FMD as compared with ARAS. A post-hoc analysis in patients with very high grade ARAS (a reduction in luminal diameter more than $70 \%$, instead of using more than $50 \%$ as the cutoff) showed similar results with regard to MRBF and renin secretion (Supplemental Table S2, http:// links.lww.com/HJH/A718). In another post-hoc analysis, we found a reduction in BP (according to the Rundback criteria) [14] 1 year after balloon angioplasty in $51 \%$ of the patients with multifocal FMD, without any differences in MRBF, renin levels, or RSR between responders and nonresponders (Supplemental Table S3, http://links.lww.com/ HJH/A718).

\section{Associations with blood pressure and renal blood flow}

As shown in Fig. 3, a positive correlation between renin levels and SBP (during 24-h ABPM) was observed in ARAS ( $\beta$ 0.257; $P=0.046$ ). Interestingly, this relationship was inverse in the FMD group $(\beta-0.531 ; P=0.002)$. As shown in Supplemental Fig. S1, http://links.lww.com/HJH/A718, 24-h urinary sodium excretion, a proxy for dietary sodium intake, was associated with MRBF in FMD ( $\beta$ 0.357; $P=0.015)$, but not in ARAS $(\beta-0.084 ; P=\mathrm{NS})$. No significant correlation was found between renin levels and MRBF. As demonstrated in Supplemental Fig. S2, http://links.lww.com/HJH/A718, there was no significant correlation between urinary sodium excretion and renin levels in either ARAS or FMD. A strong correlation was found between urinary sodium excretion and SBP (during 24-h ABPM) in the FMD group $(\beta$ 0.575; $P=0.002)$, but not in ARAS.

\section{DISCUSSION}

In this study, we evaluated renal blood flow and renin secretion, and their relation to BP in untreated patients with multifocal renovascular FMD and patients with high-grade ARAS. Our aim was to evaluate whether differences in pathophysiological mechanisms exist between FMD and ARAS, and, as our results show, this turned out to be the case.

TABLE 2. Renin levels and renovascular resistance

$\begin{array}{llcccccc}\text { Group } & & \mathbf{N} & \begin{array}{c}\text { Arterial renin } \\ \text { level (mU/I) }\end{array} & \begin{array}{c}\text { Renin (V-A)/A } \\ \text { nonaffected } \\ \text { or left kidney }\end{array} & \begin{array}{c}\text { Renin (V-A)/A } \\ \text { affected or } \\ \text { right kidney }\end{array} & \begin{array}{c}\text { RVR nonaffected } \\ \text { or left } \\ \text { kidney (AU) }\end{array} \\ \text { Unilateral } & \text { FMD } & 39 & 21.0(13.1-35.9) & 0.16(0.00-0.31) & 0.16(0.01-0.35) & 0.48(0.40-0.61) \\ \text { Rilateral } & \text { ARAS } & 63 & 19.1(11.6-45.8) & 0.08(-0.01 \text { to } 0.20) & 0.31(0.08-0.93)^{* * * *} & 0.61(0.48-0.77)^{* * *} & 0.43(0.36-0.57) \\ \text { kidney (AU) }\end{array}$

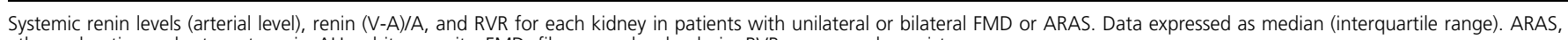

atherosclerotic renal artery stenosis; AU, arbitrary units; FMD, fibromuscular dysplasia; RVR, renovascular resistance.

${ }^{*} P=0.032$ vs. unilateral FMD.

${ }^{* *} P<0.001$ vs. contralateral kidney.

${ }^{* * *} P<0.028$ vs. FMD. No statistically significant differences were found for other between-group and within-group comparisons. 
(a)

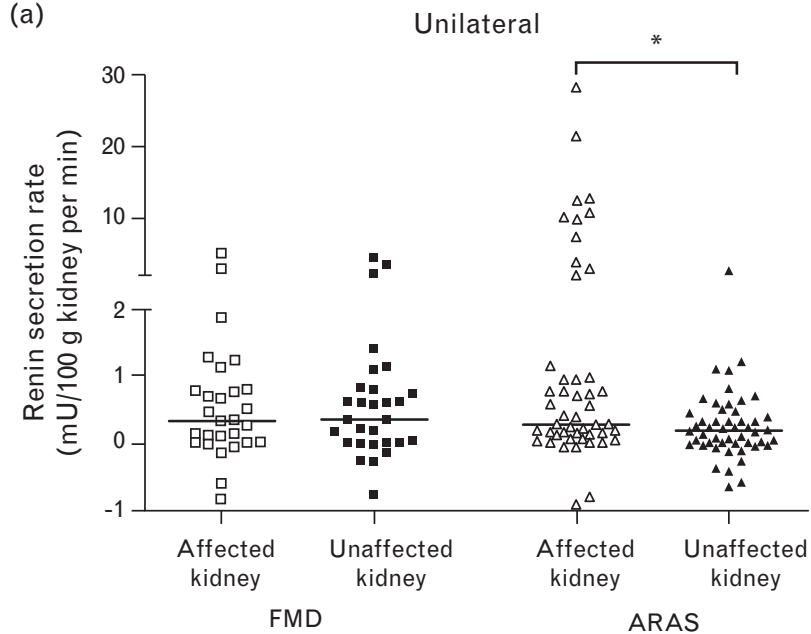

(b)

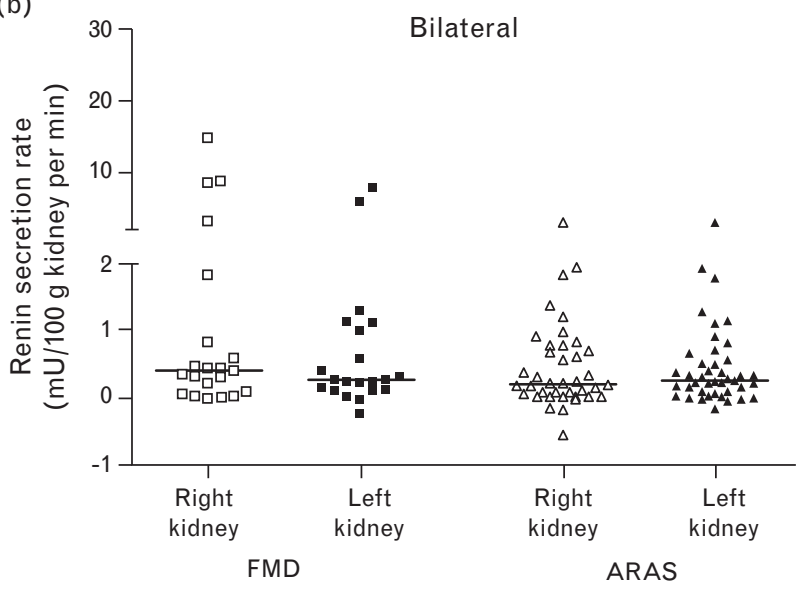

FIGURE 2 Renin secretion rate. Renin secretion rates of patients with unilateral or bilateral fibromuscular dysplasia or atherosclerotic renal artery stenosis. Horizontal line indicates median. ARAS, atherosclerotic renal artery stenosis; FMD, fibromuscular dysplasia. ${ }^{*} P=0.014$ for comparison between affected and nonaffected kidney in unilateral atherosclerotic renal artery stenosis. No statistically significant differences were found for within-group or between-group comparisons.

First, renal blood flow was higher in kidneys with FMD as compared with kidneys with ARAS. One small earlier study yielded similar results [16]. Moreover, we previously demonstrated that renal blood flow in kidneys with FMD is comparable with that in matched patients with essential hypertension [4], suggesting that renal blood flow is not substantially reduced in patients with FMD.

Second, systemic renin levels and local renin secretion were higher in kidneys with ARAS as compared with kidneys with FMD (after adjustment for differences in age, sex, and dietary sodium intake - factors that are known to influence renin levels) [17,18]. Furthermore, lateralization in renin secretion [assessed in three different ways using renal vein renin ratio, renin (V-A)/A, and RSR] was found in patients with unilateral ARAS (as expected), but not in any of the patients with unilateral FMD. Although severely elevated renin levels have been reported in patients with multifocal FMD (possibly due to more severe hemodynamic impact of the string-of-beads) [19], this appears to be more exception than rule given that none of the 64 patients in our study had elevated renin levels. Together with our previous findings that renin secretion and the response to modulation of the renin-angiotensin system in patients with FMD is comparable with that in matched patients with essential hypertension [4], the present study suggests that the presence of a 'string-ofbeads' itself does not lead to increased local renin secretion.

Third, the correlation between renin levels and BP is inverse in patients with FMD, whereas there is a direct relation between these variables in patients with ARAS. The latter is in line with the generally accepted view that hypertension in ARAS is caused by increased renin secretion [3]. In FMD, however, the correlation between renin levels and BP is inverse, similar to what is usually seen in humans without renovascular abnormalities [20]. Although we have to be cautious with drawing conclusions as these data are only cross-sectional (without information on the effects of intraindividual variation in renin secretion or BP), this suggests that systemic BP can still be transmitted to the juxtaglomerular apparatus in kidneys with FMD, resulting in lower renin secretion. In ARAS, on the other hand, RVR is higher (as shown in the present study), so that excessive renin secretion ensues from ischemic nephrons.

Taken together, these findings all argue against the generally accepted view that FMD induces hypertension (similarly to ARAS) via decreased renal blood flow and increased renin secretion. One could argue that this indicates that the FMD patients in our cohort do not have 'true' renovascular hypertension and thus would probably (a)

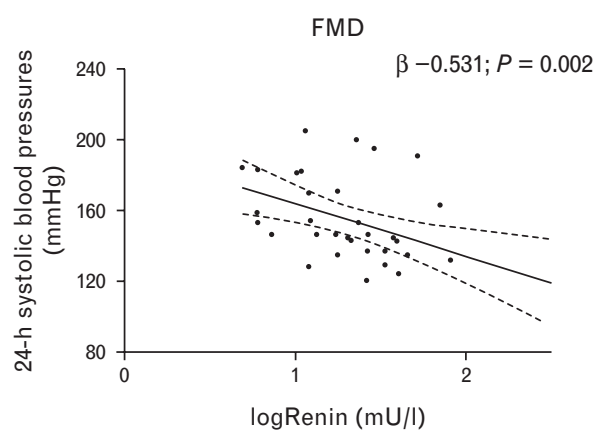

(b)

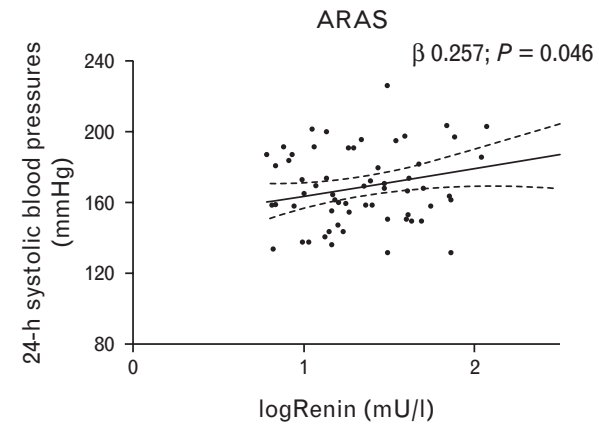

FIGURE 3 Associations between blood pressure and renin level. Correlation between SBP (during 24-h ambulatory blood pressure measurement) and renin level for fibromuscular dysplasia (a) and atherosclerotic renal artery stenosis (b). ARAS, atherosclerotic renal artery stenosis; FMD, fibromuscular dysplasia. 
not respond to revascularization. This was, however, not the case: although not designed as an intervention trial, a post-hoc analysis revealed that balloon angioplasty reduced BP (according to the Rundback criteria) [14] in $51 \%$ of the patients with multifocal FMD, indicating that at least in those patients the string-of-beads contributed to maintaining high BP. Interestingly, we observed no differences in MRBF or renin secretion between FMD patients who responded to balloon angioplasty and those who did not. Moreover, several small studies previously reported that revascularization can reduce or cure hypertension in patients with FMD without increased renin secretion $[10,11,21,22]$. Hence, renin levels should not be used as a diagnostic tool or a guide for treatment decisions in patients with multifocal FMD. Furthermore, we cannot but conclude that it is likely that the commonly held (but unproven) hypothesis, that renovascular FMD causes hypertension due to a reduction in renal perfusion and subsequently an increase in renin secretion, is false.

The question remains which pathophysiological mechanism is responsible for the development of hypertension in patients with FMD. As FMD is also found in nonhypertensive humans [23], it is very well possible that a subset of the patients with FMD dysplasia have, in fact, essential hypertension, with the string-of-beads as an innocent bystander' that does not contribute to high BP. This, however, would not explain the success of balloon angioplasty in these patients [24]. Another possibility is that the reninangiotensin system is only activated in an early stage of the disease. Longstanding exposure to an activated reninangiotensin system and high BP could have led to severe parenchymal damage, increasing BP due to increased peripheral resistance and volume overload due to excess water and sodium retention [25]. On the long term, parenchymal damage and volume overload could decrease renin-angiotensin system activity again, resulting in normal renin secretion as we observed. However, we would have expected that lateralization of renin secretion occurred in at least some patients with unilateral FMD in our cohort (i.e. those still in an early stage of the disease), which was not the case. Neither would this hypothesis fit into our previous findings that the renal microvasculature and the response to renin-angiotensin system modulation are relatively preserved in FMD [4]. Furthermore, it would not explain the success of revascularization as that would not reverse parenchymal damage already done. Other possible mechanisms include increased levels of nitric oxide inhibitors, reactive oxygen species, or other paracrine stimuli, but that has not been evaluated in FMD patients yet. Possibly, the string-of-beads induces other hemodynamic changes aside from changes in blood flow, such as changes in local pulse wave transmission, local arterial pressure, and wall stress due to increased turbulence of blood flow. These changes could act as the forces that determine the strain stimulus at the juxtaglomerular level, but apparently such stimulus does not result in increased renin secretion in FMD patients. However, it could lead to activation of other hypertensioninducing pathways, for example, to increased sympathetic nervous system activity. A report of successful renal denervation in a patient with renal artery FMD would be consistent with that hypothesis [26], but without further research we can only hypothesize on these possible mechanisms.

In addition, two other noteworthy findings were observed in this study. First, renal blood flow was lower in the left kidney as compared with the right in patients with bilateral ARAS. We previously observed this asymmetry in blood flow between both kidneys also in patients with essential hypertension [27]. Interestingly, it was not found in patients with bilateral FMD, suggesting that the flow balance between both kidneys has changed in these patients. Second, we observed in some patients with FMD or ARAS that RSR was negative in one kidney. We hypothesize that local renin production is suppressed to almost 0 in those kidneys, resulting in a negative RSR as some of the renin from the circulation is lost due to glomerular filtration [28]. The nature of these two phenomena and whether they play a role in the pathophysiology of hypertension, however, remains unknown.

The present study is limited by its small power. However, given the low incidence of diagnosed FMD in the hypertensive population, this study can still be considered relatively large. As expected, patients with FMD were younger and more often women than patients with ARAS [29,30], resulting in difficulties in comparing both groups. Although we attempted to adjust for these between-group differences using an ANCOVA model, these differences are also a reflection of normal clinical practice. We did not routinely measure trans-stenotic pressure gradients to assess hemodynamic severity of the string-of-beads as its clinical value has not been established in FMD yet. Although it has been suggested that such measurements could differentiate between patients with symptomatic FMD and those with asymptomatic FMD (who in fact have essential hypertension with the string-of-beads as an innocent bystander) [2,31], further clinical trials are needed to find out whether the efficacy of balloon angioplasty can be predicted beforehand in individual FMD patients. Although the present study was not designed to evaluate the outcome of balloon angioplasty, post-hoc analyses revealed that balloon angioplasty reduced BP in 51\% of the FMD patients in this cohort, indicating that at least in those patients the string-of-beads contributed to maintaining high BP. A relatively high number of nonresponders is to be expected in a population of this age [24], presumably due to irreversible changes in the systemic circulation caused by long-lasting hypertension. Furthermore, our FMD cohort will be relatively heterogeneous with regard to duration of hypertension and pretreatment with antihypertensive drugs. This is, however, also a reflection of normal clinical practice and inevitable in such a clinical study in newly diagnosed patients. In addition, selection or referral bias may have occurred, as we only included patients who had been referred for difficult-to-treat hypertension or clinical suspicion for renovascular abnormalities. Patients with undiagnosed FMD who responded well to antihypertensive drugs (which is probably a large number of cases given the relatively high prevalence of renovascular FMD among kidney donor candidates) [2] probably remained unrecognized and were not included in the present study.

In conclusion, we found several arguments against the hypothesis that the pathophysiological mechanisms 
leading to hypertension in patients with FMD are similar to those in patients with ARAS. Especially the findings that renal blood flow is more preserved, that local renin secretion is not increased, and that the association between renin levels and BP is inverse in patients with FMD, contradict with the commonly held but unproven hypothesis that hypertension in renovascular multifocal FMD is caused by a reduction in renal perfusion and, subsequently, increased renin secretion. Future research is needed to answer the question which alternative pathophysiological mechanisms are involved in hypertension due to renovascular FMD.

\section{ACKNOWLEDGEMENTS}

The authors wish to thank Mrs C. de Haan and Mrs M. FussLejeune for their support in performing the measurements and collecting and handling the blood samples.

\section{Conflicts of interest}

There are no conflicts of interest.

\section{REFERENCES}

1. Persu A, Giavarini A, Touzé E, Januszewicz A, Sapoval M, Azizi M, et al. European consensus on the diagnosis and management of fibromuscular dysplasia. J Hypertens 2014; 32:1367-1378.

2. Olin JW, Gornik HL, Bacharach JM, Biller J, Fine LJ, Gray BH, et al. Fibromuscular dysplasia: state of the science and critical unanswered questions: a scientific statement from the American Heart Association. Circulation 2014; 129:1048-1078.

3. Textor SC, Lerman L. Renovascular hypertension and ischemic nephropathy. Am J Hypertens 2010; 23:1159-1169.

4. van Twist DJ, Houben AJ, de Haan MW, de Leeuw PW, Kroon AA. Renal hemodynamics and renin-angiotensin system activity in humans with multifocal renal artery fibromuscular dysplasia. J Hypertens 2016; 34:1160-1169.

5. Kesteloot H, Joossens JV. The relationship between dietary intake and urinary excretion of sodium, potassium, calcium and magnesium: Belgian Interuniversity Research on Nutrition and Health. J Hum Hypertens 1990; 4:527-533.

6. Wierema TK, Houben AJ, Kroon AA, Koster D, van der Zander K, van Engelshoven JM, de Leeuw PW. Nitric oxide dependence of renal blood flow in patients with renal artery stenosis. J Am Soc Nephrol 2001; 12:1836-1843.

7. Savard S, Steichen O, Azarine A, Azizi M, Jeunemaitre X, Plouin PF. Association between 2 angiographic subtypes of renal artery fibromuscular dysplasia and clinical characteristics. Circulation 2012; 126:3062-3069.

8. Olin JW. Is fibromuscular dysplasia a single disease? Circulation 2012; 126:2925-2927.

9. Deinum J, Derkx FH, Schalekamp MA. Improved immunoradiometric assay for plasma renin. Clin Chem 1999; 45:847-854.

10. Luscher TF, Greminger P, Kuhlmann U, Siegenthaler W, Largiader F, Vetter W. Renal venous renin determinations in renovascular hypertension. Diagnostic and prognostic value in unilateral renal artery stenosis treated by surgery or percutaneous transluminal angioplasty. Nephron 1986; 44 (Suppl 1):17-24.

\section{Reviewers' Summary Evaluations \\ Reviewer 1 \\ Strengths:}

The message addressed is new for the pathophysiological implications and for the translational message in avoiding renin assessment as a guideline for the intention-to-treat
11. Luscher TF, Vetter H, Studer A, Pouliadis G, Kuhlmann U, Glänzer K, et al. Renal venous renin activity in various forms of curable renal hypertension. Clin Nephrol 1981; 15:314-320.

12. Blaufox MD, Fromowitz A, Lee HB, Mend CH, Elkin M. Renal blood flow and renin activity in renal venous blood in essential hypertension. Circ Res 1970; 27:913-920.

13. Levey AS, Stevens LA, Schmid CH, Zhang YL, Castro AF 3rd, Feldman HI, et al. A new equation to estimate glomerular filtration rate. Ann Intern Med 2009; 150:604-612.

14. Rundback JH, Sacks D, Kent KC, Cooper C, Jones D, Murphy T, et al. Guidelines for the reporting of renal artery revascularization in clinical trials. American Heart Association. Circulation 2002; 106:1572-1585.

15. WHO Collaborating Centre for Drug Statistics Methodology. Guidelines for ATC classification and DDD assignment, 2015. Oslo: WHO Collaborating Centre for Drug Statistics Methodology; 2014.

16. Lerman LO, Taler SJ, Textor SC, Sheedy PF 2nd, Stanson AW, Romero JC. Computed tomography-derived intrarenal blood flow in renovascular and essential hypertension. Kidney Int 1996; 49:846-854.

17. Hilliard LM, Sampson AK, Brown RD, Denton KM. The 'his and hers' of the renin-angiotensin system. Curr Hypertens Rep 2013; 15:71-79.

18. Weidmann P, De Myttenaere-Bursztein S, Maxwell MH, de Lima J. Effect on aging on plasma renin and aldosterone in normal man. Kidney Int 1975; 8:325-333.

19. Petruzzelli M, Taylor KP, Koo B, Brown MJ. Telling Tails: very high plasma renin levels prompt the diagnosis of renal artery stenosis, despite initial negative imaging. Hypertension 2016; 68:11-16.

20. De Leeuw P, Birkenhäger WH. Renal mechanisms of blood pressure regulation: clinical evidence. In: Zanchetti A, editor. Handbook of hypertension. Amsterdam, The Netherlands: Elsevier Science B.V.; 1997.

21. Vetter W, Vetter H, Tenschert W, Kuhlmann U, Studer A, Glänzer K, et al. Renovascular hypertension. Prognostic value of renal venous renin determinations (author's transl). Klin Wochenschr 1979; 57:863-873.

22. Marks LS, Maxwell MH, Kaufman JJ. Nonrenin-mediated renovascular hypertension: a new syndrome? Lancet 1977; 1:615-617.

23. McKenzie GA, Oderich GS, Kawashima A, Misra S. Renal artery fibromuscular dysplasia in 2,640 renal donor subjects: a CT angiography analysis. J Vasc Interv Radiol 2013; 24:1477-1480.

24. Trinquart L, Mounier-Vehier C, Sapoval M, Gagnon N, Plouin PF. Efficacy of revascularization for renal artery stenosis caused by fibromuscular dysplasia: a systematic review and meta-analysis. Hypertension 2010; 56:525-532.

25. Salem MM. Pathophysiology of hypertension in renal failure. Semin Nephrol 2002; 22:17-26.

26. Kelle S, Teller DC, Fleck E, Stawowy P. Renal denervation in fibromuscular dysplasia. BMJ Case Rep 2013. Published online: 19 August 2013.

27. van Onna M, Houben AJ, Kroon AA, Wierema TK, Koster D, van Engelshoven JM, et al. Asymmetry of renal blood flow in patients with moderate to severe hypertension. Hypertension 2003; 41:108-113.

28. Roksnoer LC, Heijnen BF, Nakano D, Peti-Peterdi J, Walsh SB, Garrelds IM, et al. On the origin of urinary renin: a translational approach. Hypertension 2016; 67:927-933.

29. Giavarini A, Savard S, Sapoval M, Plouin PF, Steichen O. Clinical management of renal artery fibromuscular dysplasia: temporal trends and outcomes. J Hypertens 2014; 32:2433-2438; Discussion 8.

30. Olin JW, Froehlich J, Gu X, Bacharach JM, Eagle K, Gray BH, et al. The United States Registry for Fibromuscular Dysplasia: results in the first 447 patients. Circulation 2012; 125:3182-3190.

31. Persu A, Van der Niepen P, Touzé E, Gevaert S, Berra E, Mace P, et al. Revisiting Fibromuscular Dysplasia: rationale of the European Fibromuscular Dysplasia Initiative. Hypertension 2016; 68:832-839.

with PTA in fibromuscular dysplasia renal artery stenosis (RSA).

Weaknesses:

Renin basal levels are in general a weak marker for assessing the significance of RAS and for providing the postinterventional outcome. The Captopril test partially overcome such poor significance. 
Authors measured renal blood flow and not the transstenotic pressure gradient. Thus, the question on which is the main hemodynamic stimulus for juxtaglomerular activation remains open.

\section{Reviewer 2}

Fibromuscular dysplasia (FMD) is one of the causes of secondary hypertension, particularly in younger women. The fact that it affects the renal arteries does not mean that it causes hypertension through the same mechanisms as atherosclerotic renal artery stenosis (ARAS). In the present paper, van Twist and colleagues studied renin levels and blood flow in patients with FMD and ARAS and found differences in these parameters and how renin relates to blood pressure. Further data especially on all components of the renin-angiotensin-aldosterone system and better characterization of the hemodynamic effects of structural changes of the renal arteries are required to fully understand the pathophysiology of hypertension in patients with FMD. The present paper is, however, an important first step. 